\title{
Experimental Study on the Circular Loop Antenna Immersed Shallowly in a Conducting Medium ${ }^{1}$
}

\author{
Keigo Iizuka
}

\author{
Division of Engineering and Applied Physics, Harvard University, Cambridge, Mass.
}

(Received December 21, 1964; revised March 18, 1965)

\begin{abstract}
The behavior of a circular loop antenna near the interface between a conducting medium and air has been studied experimentally in terms of the following several parameters:

(1) Circumference per wave length $\beta b$ of the loop

(2) Loss tangent of the conducting medium

(3) Depth of the loop below the interface

(4) Angle between the plane of the loop and the interface.

Comparisons were made of the behavior of a loop when oriented both parallel to the interface and perpendicular to it in terms of such quantities as the driving-point admittance, the amplitude and phase distributions of the current, the effect of varying the distance from the interface, and the upward transmission of power into air from the loop when immersed in the conducting solution.

It was found that as the depth of the loop is increased the values of the driving-point admittance asymptotically approach the theoretical results by King, Harrison, and Tingley [1964] and Wu [1962] for an infinite medium. As the size of the loop became larger the difference between the driving-point admittance for the loop oriented parallel to the interface and that for the loop perpendicular to the interface increased. It was discovered that there exists an optimum angle tilted from either parallel or perpendicular orientation for the best upward transmission of power.
\end{abstract}

\section{Introduction}

The present study extends earlier work on a loop in an infinite homogeneous medium to a loop near the interface between two semi-infinite media. Evidently, the half-space configuration is of practical significance, since it approximates such situations as antennas under a landing strip for aircraft, submarine antennas, missile antennas in plasmas, or buried antennas for survival telecommunications.

To the best of the author's knowledge, however, no theoretical analysis of a circular loop of finite size located near the interface between a dissipative medium and free space has been reported. This problem is mathematically complex, owing to the boundary conditions along the interface. Hence, an experimental investigation of this subject has a special significance.

Experiments were performed to determine the driving-point admittance, current amplitude, and phase distributions, and radiated power for a loop when immersed in a dissipative medium at an arbitrary orientation near a plane interface. The parameters used and their ranges are summarized as follows:

(1) Circumference per wavelength of the loop $\beta b=\frac{2 \pi}{\lambda} b=0.4$ to 1.2 ; ${ }^{1}$ This research was supported by Air Force Cambridge Research Laboratories, Office of
Aerospace Research, Contract AF 19(604)-7262 and National Science Foundation Grant $\mathrm{G}-20225$.
(2) Loss tangent of the medium, $\frac{\sigma}{\omega \epsilon_{r} \epsilon_{0}}=0.036$ to 2.64;

(3) Distance of the loop from the interface, $d$ $=\frac{\lambda}{2 \pi}$ to $2 \lambda \sim \infty$;

(4) Angle $\theta$ between the plane of the loop and the plane perpendicular to the interface, $\theta=0$ to $180^{\circ}$;

(5) Angle $\Phi$ between the line which connects the driving-point and the center of a loop and that which connects a point on the circumference and the center of the loop, $\Phi=0$ to $180^{\circ}$.

Consideration is given to the question: which of the parallel and perpendicular orientations of the loop with respect to the interface are advantageous from the viewpoint of frequency bandwidth, insensitivity to changes in the surrounding medium, and upward transmission of the power. It may be added that it was discovered that neither parallel nor perpendicular orientation is the best for the transmission of power from the loop in the solution into the air above it.

\section{Experimental Arrangement}

The previously described setup designed for the study of antennas in a homogeneous dissipative medium [Iizuka, 1961, 1962, 1965] was used with certain modification. A detailed description of the apparatus is given in the earlier reports. A water solution of sodium chloride was used to fill partially a tank having dimensions $4 \frac{1}{2} \lambda \times 4 \frac{1}{2} \lambda \times 25 / 8 \lambda$, where $\lambda=29.77$ 
$\mathrm{cm}$ is the wavelength in the water solution, $\frac{\sigma}{\omega \epsilon_{r} \epsilon_{0}}$ $=0.036, \epsilon_{r}=78$, and the corresponding frequency is $114 \mathrm{Mc} / \mathrm{s}$. The wavelength used is always that in the conducting medium. As shown in figure 1 , five of the six walls are made of wood, and the sixth, arranged in a vertical position, is an aluminum image plane. The semicircular loop was installed over the image plane and driven by a slotted line. A receiving antenna was installed in the air exactly above the driving-point of the loop in order to measure the power radiated upward above the interface as a function of the orientation and size of the loop. By measuring the standing wave pattern on the feeding coaxial line, the power $P_{t}$ $\left(\sim E_{\max } \cdot E_{\min }\right)$ transferred outward from the antenna was determined. The loop antenna was slotted along the circumference so that a small shielded current probe could be moved along the circumference by pulling the attached cable by means of a carriage on a rack outside the tank. The probe was covered by slotted plastic tubing and had no direct contact with the solution. This construction was necessary so that the conducting solution would not short-circuit the opening of the shielded probe.

The loop was clipped onto the inner conductor of the line with a brass-fingered connector, and the other end of the circular loop was pressed against the aluminum image plane, which was moistened with silver paint.
The loops were made of iron, and the attractive force of a strong permanent magnet installed outside the tank directly opposite the loop assured a good electrical contact with the image plane and facilitated changes in the orientation of the loop.

\section{Driving-Point Admittance}

The driving-point admittance of the loop was measured repeatedly with the same solution at different levels by draining out measured quantities of the solution.

In figure 2 the measured driving-point admittances of the loop immersed in a solution with the loss tangent $\frac{\sigma}{\omega \epsilon_{r} \epsilon_{0}}=0.1, \epsilon_{r}=78$ at the depth $d / \lambda=0.125$ are compared in the complex $G-B$ plane with that of the loop immersed in the same solution but infinitely far from the interface (in broken line). The normalized circumference $\beta b$ of the loop is the parameter. One of the two curves shown in solid lines is for the loop oriented parallel to the interface, and the other is for the loop perpendicular to the interface. As the size of the loop becomes larger, the difference between the driving-point admittance for the loop oriented parallel to the interface and that for the loop perpendicular to the interface is increased. Note that for the larger

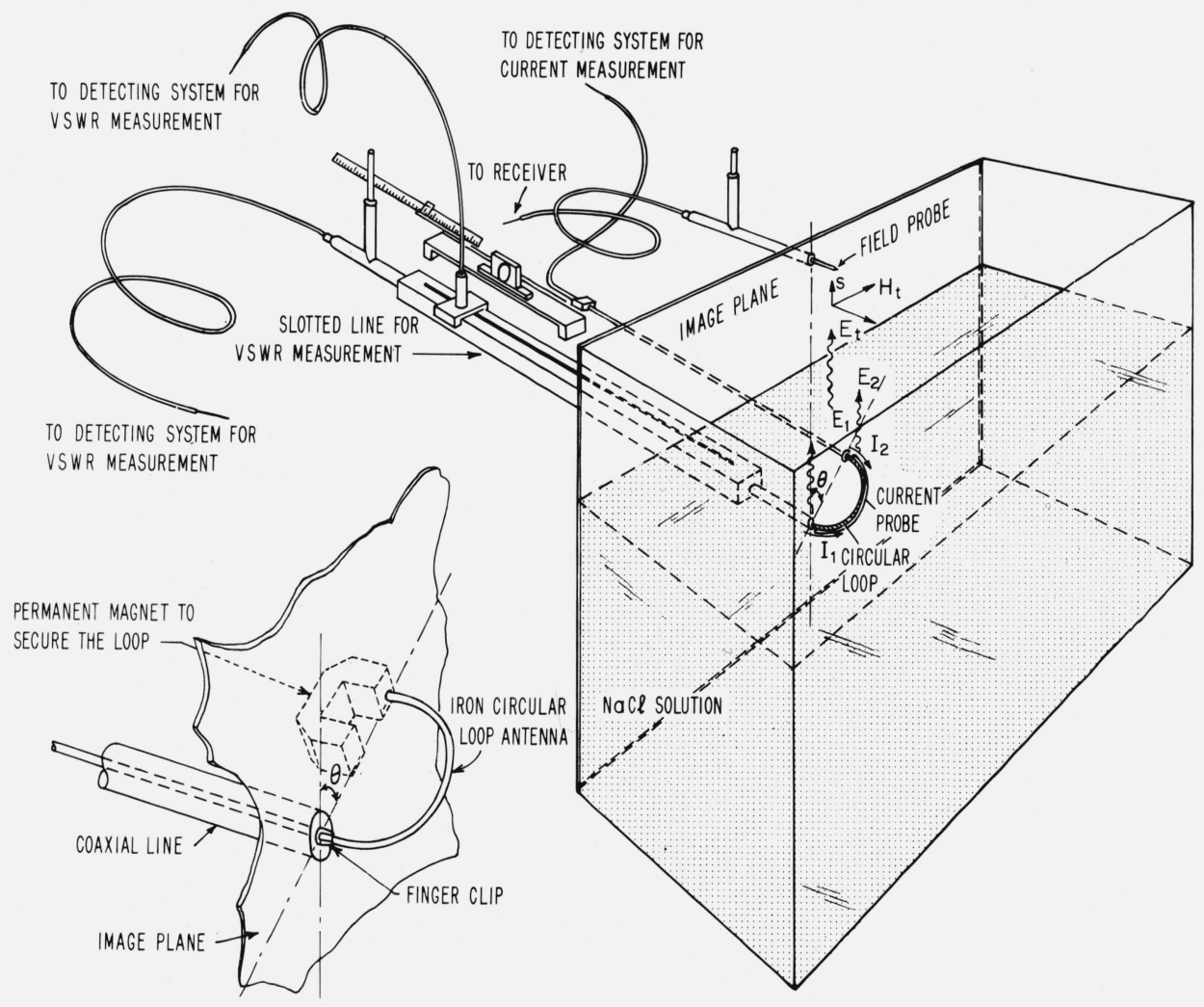

FIGURE 1. Block diagram of experimental equipment. 
loops the curves for perpendicular orientation differ less from that for an infinite medium than does the curve for parallel orientation. Since the main radiation lobe of the larger loop $(\beta b=1.0$ and 1.2) excited principally in the dipole mode is in the direction perpendicular to the plane of the loop, the interface is out of the direction of the main lobe when the plane of the antenna is oriented perpendicular to the interface. And also, the average depth of the perpendicular loop is greater than that for the parallel loop. As a result, the interaction of the perpendicular loop with the interface is smaller, and a smaller deviation from the admittance of the loop in an infinite medium occurs.

The experiment was repeated with a much smaller depth of the loop at $d / \lambda=0.037$. This is the depth at which the entire base of the coaxial feeding line was barely immersed in the solution, i.e., $d=B\left(B=7 / 16^{\prime \prime}\right)$ in the end view of the base of the antenna driven by a coaxial line shown in figure 2 . The measured results with the smaller depth are indicated by crosses with an arrow. It is seen that the spiral shapes of the curves for either orientation were preserved but became smaller in size with a decrease in depth. For a loop with smaller values of $\beta b$, the changes in the driving-point admittance due to the reduction in depth are less significant than for those with larger values of $\beta b$.

To see the effect of the depth of a loop on the drivingpoint admittance, the measurements were made at intermediate values of $d / \lambda$ with various orientations in the solution with $\frac{\sigma}{\omega \epsilon_{r} \epsilon_{0}}=0.1$ and $\epsilon_{r}=78$. The results are shown in figure 3 as a function of $d / \lambda$. The sizes of the loops used were $\beta b=0.4,0.6$, and 1.0 in three different orientations. They are:

(1) parallel to the interface, denoted by solid dots in the figure;

(2) perpendicular to and with the driving-point of the antenna nearest the interface, denoted by small circles; and

(3) perpendicular to the interface with the drivingpoint at the deepest point, denoted by triangular points. With configuration 3 , the measurements were made only with a loop with $\beta b=0.6$. The upper end of the loop broke the interface in a range of the depth smaller than $0.191 \lambda$.

It is seen that as the depth $d / \lambda$ increases both the conductance and susceptance curves become flatter and asymptotically approach the values for a circular loop antenna immersed in an infinite dissipative medium. The curves for the loops with $\beta b=0.4$ and 0.6 and with any orientation are flattened faster (a smaller value of $d / \lambda$ ) than those for the loop with $\beta b=1.0$ in any orientation. The corresponding values calculated by King, Harrison, and Tingley [1964] based on Wu's [1962] theory for an infinite medium are included in figure 3 . (The measured values of the driving-point impedances are for a half loop over a conducting plane so that the theoretical admittance of the corresponding loop must be multiplied by two.) The curves for the loop with $\beta b=1.0$ in the perpendicular orientation are flattened faster than those in the

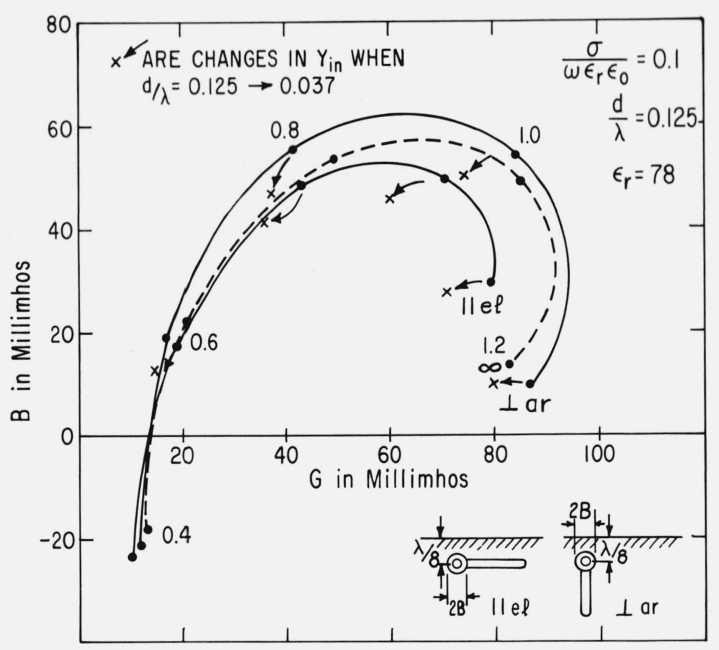

FIGURE 2. Driving-point admittances of the loop near and parallel or perpendicular to the interface between air and a dissipative

medium with $\frac{\sigma}{\omega \epsilon_{\mathrm{r}} \epsilon_{0}}=0.1$ and $\epsilon_{\mathrm{r}}=78$.

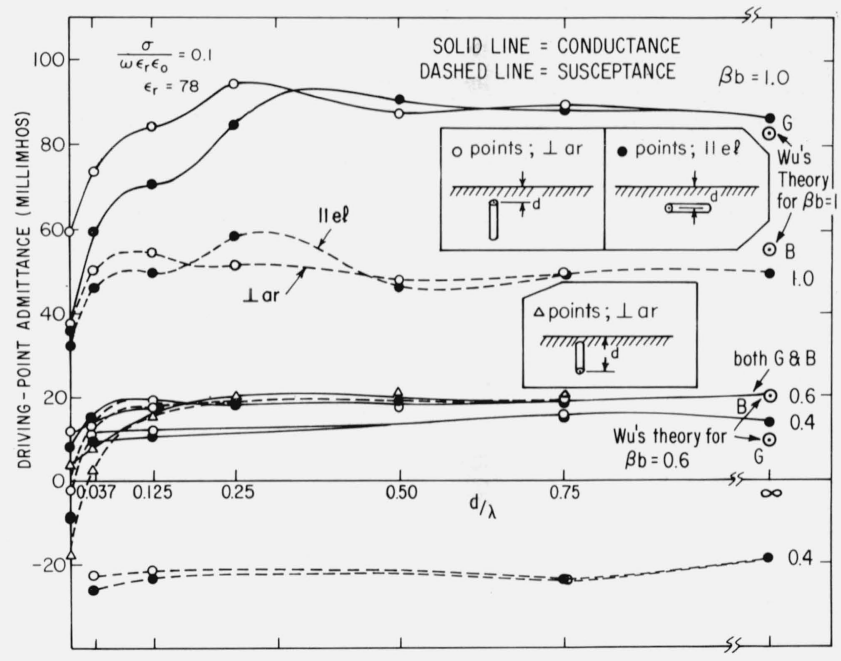

FIGURE 3. Driving-point admittance of the loops either parallel or perpendicular to the interface as a function of the depth $\mathrm{d} / \lambda$ of the loop.

horizontal orientation. This may be not only because the average depth is larger but also because the direction of the main lobe of a loop in the former orientation is $90^{\circ}$ from the direction of the interface, whereas in the latter it is in the direction of the interface. Hence, it may be said that in case a knowledge of the characteristics of a circular loop immersed in such a medium is to be utilized in determining the properties of the medium, the perpendicular orientation of the loop requires the least depth of the driving-point of the loop from the interface.

Observe that a rather marked kink appears in the curves near $d / \lambda=0.125$ (recall that $d / \lambda=0.191$ is the depth at which the entire loop of size $\beta b=0.6$ is barely immersed in the solution when the loop is in 
the previously mentioned configuration 3 ) in the curves of both $G$ and $B$ for a loop with $\beta b=0.6$ perpendicular to the interface with the driving-point at the deepest part of the loop. This illustrates that as soon as any portion of the loop is exposed above the surface of the solution, the conductance curve, and especially the susceptance curve, begin to behave quite differently from those of a loop that is entirely immersed.

It should be added that the points obtained with $d / \lambda=0$ are complicated by the fact that [Iizuka, 1964] one half of the end surface of the feeding line is in air, while the other half is immersed in the solution, so that the reactive end and coupling effects near the base of the antenna are different from those when the base is fully immersed in the solution. In addition, the dimensions of the present tank are too small to permit accurate measurements, since the wavelength outside the solution is about nine times as long as that in the solution $\left(\epsilon_{r}=78\right)$.

In figure 4 is plotted the driving-point admittance of a loop with $\beta b=1.0$ when it is either prependicular or parallel to the interface as a function of the depth $d / \lambda$. In this figure the loss tangent $\frac{\sigma}{\omega \epsilon_{r} \epsilon_{0}}$ of the solution was taken as the parameter, but in figure 3 the size of the loop was taken as the parameter. The curves for solutions with larger loss tangents are flattened in shorter distances $d / \lambda$ than those for solutions with smaller loss tangents, but regardless of how large the

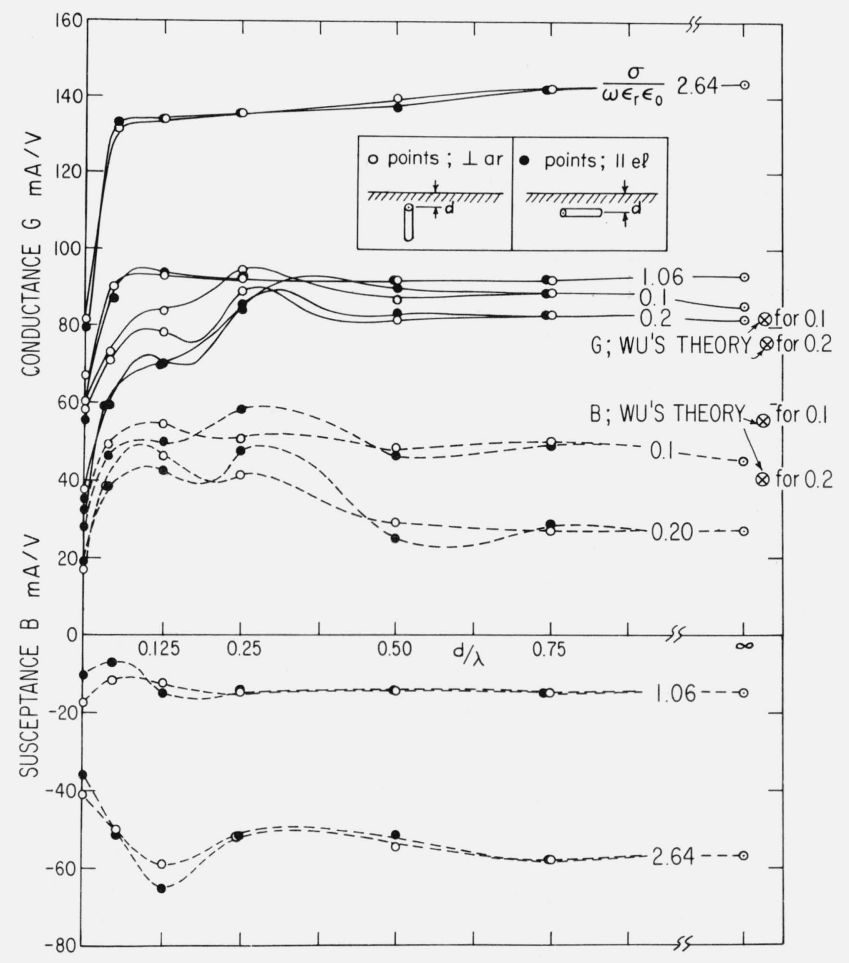

FIGURE 4. Driving-point conductance (solid line) and susceptance (dash line) of a loop with $\beta \mathrm{b}=1.0$ as a function of the depth with $\frac{\sigma}{\omega \epsilon_{\mathrm{r}} \epsilon_{0}}$ as a parameter. loss tangent may be, oscillations in the amplitude are observed when $d / \lambda$ is smaller than 0.15 or 0.2 . In this sense $d / \lambda=0.15 \sim 0.20$ may be considered to be a critical depth. (It should be mentioned that the discussion here is made in terms of $d / \lambda$; note that $\lambda$ decreases as $\frac{\sigma}{\omega \epsilon_{r} \epsilon_{0}}$ is increased. It follows that the actual depth $d$ for a fixed value of $d / \lambda$ also decreases with an increase in $\frac{\sigma}{\omega \epsilon_{r} \epsilon_{0}}$, and for the limiting case $d$ approaches zero as $\frac{\sigma}{\omega \epsilon_{r} \epsilon_{0}}$ approaches infinity. In a similar way, even if the radius of the loop is fixed, the value of $\beta b$ increases with an increase in $\frac{\sigma}{\omega \epsilon_{r} \epsilon_{0}}$. In the case of the highest loss tangent, $\frac{\sigma}{\omega \epsilon_{r} \epsilon_{0}}=2.64$ in figure 4 , the value of $\beta b$ which corresponds to $\beta_{0} b=1.0$ in the solution with $\epsilon_{r}=78, \frac{\sigma}{\omega \epsilon_{r} \epsilon_{0}}=0.036$ is 1.30 ; however, for the rest of the values of $\frac{\sigma}{\omega \epsilon_{r} \epsilon_{0}}$ in the figure, $\beta b$ can be considered to be identical with $\beta_{0} b$.) It is interesting to know that the critical depth for a dipole antenna was also the same $d / \lambda=0.15 \sim 0.20$ [Iizuka, 1964].

\section{Current Amplitude and Phase Distribu- tions on the Loop}

Measurements were made of the amplitude and relative phase distributions of the current on a loop with $\beta b=1.0$ when oriented either parallel or perpendicular to the interface in a solution with $\epsilon_{r}=78, \frac{\sigma}{\omega \epsilon_{r} \epsilon_{0}}=0.2$, and with the loop at various depths. In figure $5 \mathrm{a}$ the following are shown with parallel orientation of the loop: the amplitude of the current $I(\Phi)$ along the circumference of the loop normalized to the value $Y=\frac{I(0)}{V} \mathrm{~mA} / \mathrm{V}$, and the phase $\theta_{I}$ of the current along the circumference of the loop referred to $\theta_{I}(0)=\tan ^{-1}$ $\frac{G}{B}$. In figure $5 b$, the same quantities are shown but with perpendicular orientation. All the quantities are shown as a function of $\Phi$. The curves for $d / \lambda$ $=0.0$ are for the case when the center line of the wire of the loop is exactly in the interface (disregarding a slight rise in the level of the solution due to surface tension). The curves for $d / \lambda=-0.022$ and $-\frac{1}{2 \pi}$ are, respectively, for the cases when the driving-point of the loop is located at the distance $2 a$ above the surface of the solution (where $a$ is the radius of the wire of the loop and $a=1 / 8$ in.) and when the center of the circle of the loop is located at the interface and only half of the circumference is immersed in the solution.

Note that the minimum of the magnitude of the current which exists near $\Phi=70^{\circ}$ for both parallel 

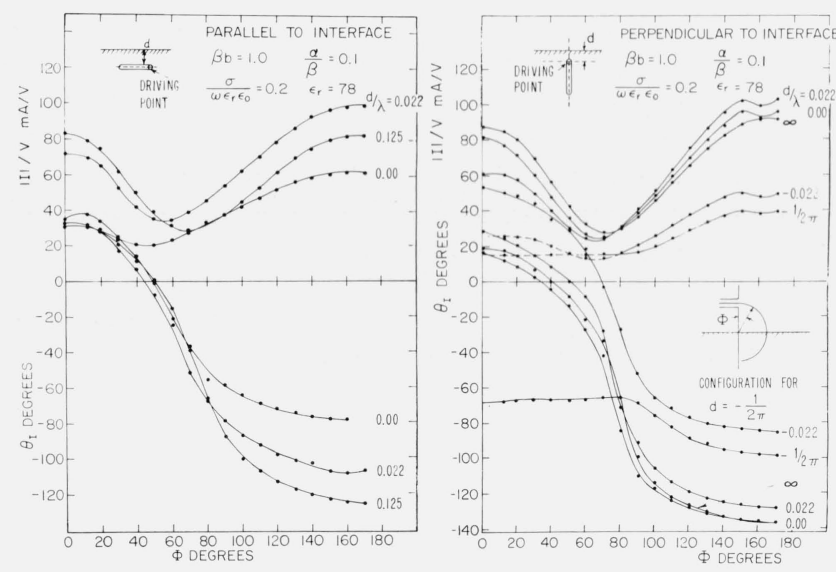

Figure 5a. Current distribution $\frac{\mathrm{I}}{\mathrm{V}}=\frac{|\mathrm{I}|}{\mathrm{V}} \epsilon^{j \theta_{\mathrm{I}}}$ along circular loop $\beta \mathrm{b}=1.0$ parallel to interface in a solution $\frac{\sigma}{\omega \epsilon_{\mathrm{r}} \epsilon_{0}}=0.2, \epsilon_{\mathrm{r}}=78$, at various depth $\mathrm{d}$ of the loop.

Figure 5b. Like figure $5 a$ but with loop perpendicular to the interface.

and perpendicular cases moves toward smaller values of $\Phi$, that the ratio of the maximum magnitude to the minimum decreases, and that the slope of the $\theta_{I}$ curve as a function of $\Phi$ becomes less steep as the antenna is moved toward the interface. In other words, the nearer the loop is to the interface, the more the distribution of current tends to resemble the distribution in a loop of smaller size. The current distributions on the perpendicularly oriented loop are much less influenced by the depth of the loop. For both orientations the distribution of the phase is more sensitive to a change in the depth than is the amplitude of the current.

The distributions of the amplitude and phase on the loop, when only half of its circumference is immersed in the solution (the curves with $d / \lambda=-\frac{1}{2 \pi}$ ), show a particularly interesting behavior. The shapes of the curves both for the amplitude and phase along the upper half of the circumference (which is in the air) are quite different from those along the lower half of the circumference which is in the solution. (No account is taken of the change in size of the current probe in terms of wavelength when it is inside and when it is outside the solution.) The former is concave upward and resembles the rest of the curves which are for loops fully immersed in the solution and the latter is flat and resembles those of a small isolated loop in air. This is due to the fact that the wavelength outside the solution is about nine times that in the solution $\left(\epsilon_{r}=78\right)$. The distribution of current in one part of an antenna is usually closely related to that in another, and a change in the distribution in one part significantly influences the distribution on other parts. In the present case, however, one loop antenna has the characteristics both of an antenna immersed in a medium and in free space with a marked border line. This means that the mutual interaction of the current elements above and in a dissipative medium is small.

\section{Upward Transmission of Power}

It is of practical interest to study the relation between the upward radiated power from a loop and the angle $\theta$ of orientation of the loop with respect to the interface. The relative power $P$ received by a monopole located in the near field perpendicular to the image plane exactly above the driving-point of the loop was measured and divided by the input power $P_{t}$ of the loop. Measurements were repeated for loops

with $\beta b=0.4,0.8$, and 1.0 in the solution with $\frac{\sigma}{\omega \epsilon_{r} \epsilon_{0}}$ $=0.2, \epsilon_{r}=78$. The height of the solution was adjusted each time so that the distance between the point of the loop nearest the interface when $\theta=0$ and the interface was always $0.13 \lambda$ (see the configuration in fig. 6). The adjusted distances $d$ from the driving-point to the surface of the solution were $d=0.26 \lambda$ for $\beta b$ $=0.4, d=0.38 \lambda$ for $\beta b=0.8$, and $d=0.45 \lambda$ for $\beta b=1.0$. In figure 6 , measured values of the relative power (linear scale) received by the monopole and the driving-

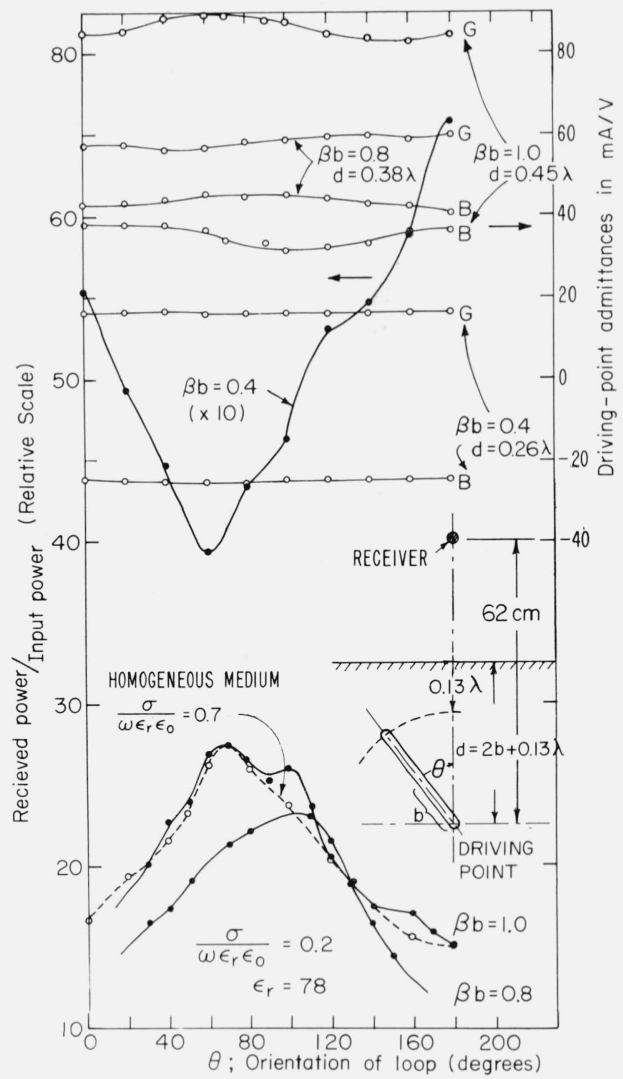

FIGURE 6. Upward transmission of power from the loop near the interface as a function of its orienting angle $\theta$. 
point admittance of the loop are shown as a function of the orientation angle of the loop. The values for $\beta b=0.4$ were multiplied by a factor of 10 for clarity. Note that in general neither the parallel nor the perpendicular orientation of the loop is best for the upward transmission of power. There exists an optimum angle slightly tilted from either parallel or perpendicular orientation. The angles of optimum transmission are 66 degrees for $\beta b=1.0,104$ degrees for $\beta b=0.8$, and 180 degrees for $\beta b=0.4$. The optimum angles for the larger loops differ significantly from either the parallel or perpendicular orientation. In general, as the size of the loop is decreased the loop should be tilted with a decreasing angle and should come close to the perpendicular orientation for $\beta b=0.4$.

The field patterns of a loop immersed in an infinite homogeneous conducting medium measured at a much larger distance have been reported [Iizuka, 1965]. The field pattern of the loop with $\beta b=1.0$ in a solution

$\frac{\sigma}{\omega \epsilon_{r} \epsilon_{0}}=0.7, \epsilon_{r}=78$, in this reference was copied into figure 6 for comparison. It was normalized to the peak of the curve for the loop with $\beta b=1.0$ near the interface between the solution with $\frac{\sigma}{\omega \epsilon_{r} \epsilon_{0}}=0.2$ and air. (The radiation pattern as a function of the loss tangent of the solution has been reported [Iizuka, 1965]. After a study of these results it is found that the curve for $\frac{\sigma}{\omega \epsilon_{r} \epsilon_{0}}=0.7$, which is the closest to the present value of $\frac{\omega \epsilon_{r} \epsilon_{0}}{\omega \epsilon_{r} \epsilon_{0}}=0.2$, can be used with a reasonably small error.)
Note that very close agreement has been obtained between the field pattern of the loop in an infinite medium and that of the corresponding loop located near the interface, except that for the latter the curves have two small humps at $\theta=100^{\circ}$ and $155^{\circ}$.

It may be concluded that the general behavior of the radiation pattern of a loop near the interface between a dissipative medium with moderate conductivity and air is not very different from that of a loop immersed in an infinite conducting medium.

The author is indebted to Professor R. W. P. King of Harvard University for correcting the manuscript.

\section{References}

Iizuka, K., and R. W. P. King (May 1961), Apparatus for the study of the properties of antennas in a conducting medium, Sci. Rept. No. 1, Cruft Lab., Harvard Univ., Cambridge, Mass.

Iizuka, K., and R. W. P. King (July 1962), The dipole antenna immersed in a homogeneous conducting medium, IRE Trans. Ant. Prop. AP-10, 384-392.

Iizuka, K. (Jan. 1964), An experimental investigation on the behavior of the dipole antenna near the interface between the conducting medium and free space, IEEE Trans. Ant. Prop. AP-12, 27-35.

Iizuka, K. (Jan. 1965), The circular loop antenna immersed in a dissipative medium, IEEE Trans. Ant. Prop. AP-13, 43-47.

King, R. W. P., C. W. Harrison, Jr., and D. G. Tingley (July 1964), The admittance of bare circular loop antennas in a dissipative medium, IEEE Trans. Ant. Prop. AP-12, 434-438.

Wu, T. T. (Nov.-Dec. 1962), Theory of thin circular loop antennas, J. Math. Phys. 3, 1301-1304.

(Paper 69D9-559) 